\title{
Access Network Selection Algorithm in Heterogeneous Multi-cognitive Wireless Networks Coexistence Environment
}

\author{
Tao Tang \\ Chongqing Information \& Communication Branch \\ Company \\ College of Communication Engineering \\ Chongqing University \\ Chongqing, China \\ E-mail: toytao@163.com \\ Yina Deng * \\ College of Communication Engineering \\ Chongqing University \\ Chongqing, China \\ E-mail: yn.deng@foxmail.com \\ * Corresponding Author
}

\author{
Weilong $\mathrm{Wu}$ \\ Chongqing Information \& Communication Branch \\ Company \\ Chongqing, China \\ E-mail: wuweilong@sina.com
}

\author{
Wenjiang Feng \\ College of Communication Engineering \\ Chongqing University \\ Chongqing, China \\ E-mail: fengwj@cqu.edu.cn
}

\begin{abstract}
Considering the three factors of user's preference, business requirement and network performance, this paper presented an integrated weighting network access selection algorithm under heterogeneous multi-cognitive wireless network coexistence environment. The algorithm based on AHP-TOPS IS algorithm which defined positive and negative ideal values, and used the distance value between the candidate networks to avoid the ranking abnormality problem when executing the TOPS IS algorithm. The algorithm introduced entropy as objective weight for decision attributes, to avoid the subjective and arbitrary problem when performing the AHP algorithm. And the algorithm used the relative relationship of the decision attributes, business types, and the network performance to define the subjective weight, and then combine the objective weight with the subjective weight to form an integrated weight. Adopting the algorithm to execute optimal access network selection can balance the network loads, and use network resources reasonably. Simulation results showed that the proposed integrated weighting network access selection algorithm can select candidate network correctly depending on the different business needs in the heterogeneous multi-cognitive wireless network coexistence environment, and can meet the service quality while achieving the entire network load balancing, and improving resource utilization.
\end{abstract}

Keywords-Heterogeneous Multi-Cognitive Wireless Networks; Access Network Selection; Analytic Hierarchy Process; Technique for Order Preference by Similarity to An Ideal Solution;

\section{INTRODUCTION}

Because of contradictions between business requirement and resource scarcity, a wireless communication network must seek new available spectrum opportunity. Cognitive radio (CR) with the characteristic of environmental awareness, behavioral awareness and purpose sense [1] allow the multi-dimensional spectrum share in the time domain, frequency domain and airspace. With the development of television, digital television has taken the place of analog television, licensed television bands left a lot blank fragment (television white space, TVWS), and TVWS band has good propagation characteristic. A variety of based cognitive radio access TVWS band communications standards have been released so far, such as IEEE802.22 [2], IEEE802.11af [3] and ECMA392 [4] and so on. From the user's perspective, the mode of heterogeneous multi-network coexistence can support multi-mode intelligent terminal to access any network coverage area, and provide better communication services for users. However, different networks have different architectures and qualities of service, in order to select the best network to access. Terminals need to estimate each network performance according to their own requirements.

The reasons triggering network selection include: different networks use different standards and they have different transmission characteristics and QoS supporting capabilities, business types have broken the traditional voice and data services division, and QoS demands have great diversity, terminals having different transmission support capabilities, business support capabilities, interactive capabilities, and great diversity of userneeds.

Existing network selection algorithms mainly consist of game theory [5-6], genetic algorith $\mathrm{m}$ [7], multi-attribute decision making (MADM) [8], fuzzy logic [9], the utility function [10] and other methods and models [11], In [6], the author proposed a cost-effective network selection algorithm aimed at to maximize user's profit, explained the relationship between operators and users with non- 
cooperative game in the abstract, chose the best network by seeking a Nash equilibrium. The authors in [9] adopted the received signal strength, available bandwidth, price and user's preference as network selection index, used fuzzy logic to calculate candidate network performance and execute the best network selection. The network selecting algorithm of the authors in [12] combined with AHP (analytic hierarchy process) and SAW (simple add weight) to perform network selection, namely used AHP computing network selection decision attribute weights, reused SAW sorting the candidate network, and then selected the best network, the authors in [13] through calculating the network performance parameters and traffic blocking probability, selected and assessed network with user's satisfaction dynamically. The authors in [14-15] based on AHP and TOPSIS (technique for order preference by similarity to an ideal solution), used AHP computing the relative importance of network selection decision attribute, reused TOPSIS executing the best network selection. The algorithm considered the received signal strength, available bandwidth, transmission speed, load factor, power consumption and price as decision attributes. QoS-based network selection algorithm, possessed small cost, good effect characteristics, but in a heterogeneous multi-network coexistence environment, while competing with the network provide the same service, the global performance will decrease, and because different network connectivity and coverage change dynamically, researchers cannot select the network just based on the quality of experience (QoE), therefore, the author in [16] presented a combination of QoS and QoE collaborative network selection method aimed at heterogeneous multi-network coexistence environment, because of network selection in the heterogeneous multinetwork coexistence environment affected by multiple factors, purely consider about users' subjective preference and QoS requirements of different services, or only pay attention to the whole performance and load balancing of network have a certain one-sidedness, it is difficult to ensure that the performance of users and operators reached the best point at the same time.

In this paper, considering the user's preference, business requirement and network performance, researchers proposed an integrated weighting network access selection algorithm for heterogeneous multicognitive wireless networks coexistence environment. Simu lation results showed that the proposed algorithm can be more awareness of the terminals' different business needs to rank and select the candidate networks reasonably in heterogeneous multi-cognitive wireless networks coexistence environment, can meet the quality of the service and achieve whole network load balancing at the same time, the algorithm improved resource utilization as a consequence.

\section{SYSTEM MODEL}

Fig. 1 showed a heterogeneous multi-cognitive wireless networks coexistence model, constructed by IEEE 802.11af Wireless local access network (WLAN), the ECMA 392 intelligent Information Grid network (IIGN), the IEEE 802.22 wire less region access network (WRAN) and TV broadcast tower.

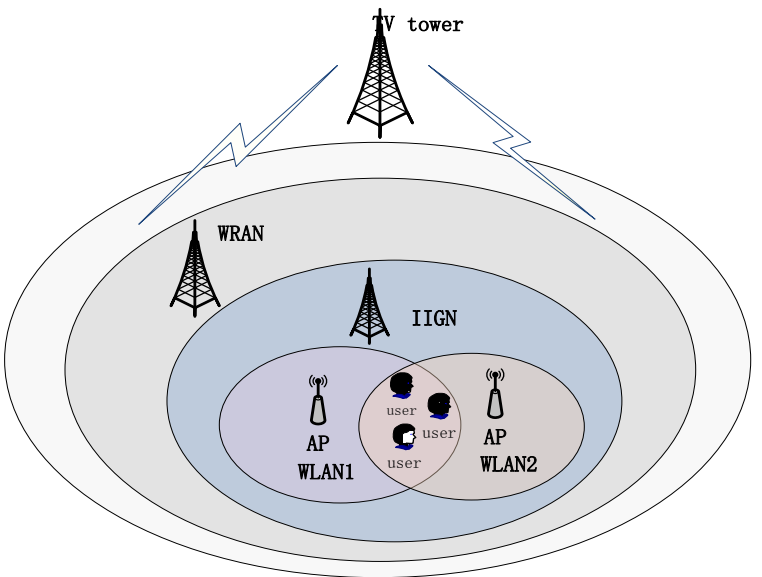

Figure 1. Heterogeneous multi-cognitive wireless networks coexistence model.

\section{INTEGRATED WEIGHTING NETWORK ACCESS SELECTION ALGORIT HM}

Step 1: To construct the decision matrix D

$$
\mathbf{D}=\left[\begin{array}{cccc}
d_{11} & d_{12} & \cdots & d_{1 n} \\
d_{21} & d_{22} & \cdots & d_{2 n} \\
\vdots & \vdots & \ddots & \vdots \\
d_{m 1} & d_{m 2} & \cdots & d_{m n}
\end{array}\right]
$$

Noted that $m$ is the number of candidate networks, $n$ is the number of decision attributes, $d_{i j}(i=1,2, \cdots, m ; j=1,2, \cdots, n)$ represents the $j_{\text {th }}$ attribute value of $i_{\text {th }}$ candidate network.

Step 2: Used the decision matrix $\mathbf{D}$ constructed by step 1 to generate normalized decision matrix $\mathbf{A}$

$$
\mathbf{A}=\left[\begin{array}{cccc}
a_{11} & a_{12} & \cdots & a_{1 n} \\
a_{21} & a_{22} & \cdots & a_{2 n} \\
\vdots & \vdots & \ddots & \vdots \\
a_{m 1} & a_{m 2} & \cdots & a_{m n}
\end{array}\right]
$$

Where

$$
a_{i j}=d_{i j} / \sqrt{\sum d_{i j}^{2}}, i=1,2, \cdots, m ; j=1,2, \cdots, n
$$

Step 3: Constructed a hierarchical structure model, and assigned each decision attribute subjectively based on business type, user's preference and network performance. The model of hierarchical structure showed in Fig. 2, where the highest layer is the best network, the second layer is the decision attributes, this paper chose delay, packet loss, jitter, speed, load and cost as the decision attributes when select networks. The lowest layer is the candidate networks. The requirement of network performance for different business types showed in TABLE 1 . Where, $\mathrm{H}$ represents high, $\mathrm{M}$ represents middle, and $\mathrm{L}$ represents low. Depending on the relative importance of each attribute calculate subjective decision weights. 


$$
\varpi_{j}^{S}=\sqrt[n]{\prod_{i=1}^{n} \delta_{i j}}, i, j=1,2, \cdots, n
$$

Where, is the relative relationship of different business types. Normalize the subjective weight $\varpi_{j}^{\dagger} \delta$ get $\omega_{j}^{S}$

$$
\omega_{j}^{S}=\varpi_{j}^{S} / \sum_{j=1}^{n} \varpi_{j}^{S}, j=1,2, \cdots, n
$$

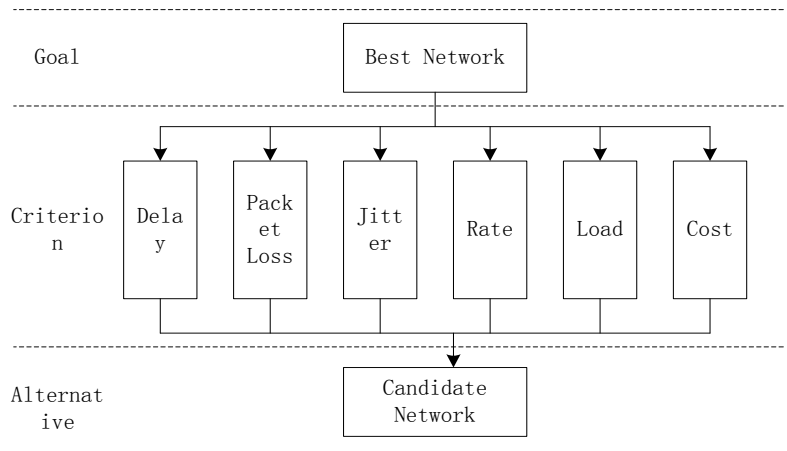

Figure 2. The model of hierarchical structure

TABLE I. THE REQUIREMENT OF NETWORK PERFORMANCE FOR DIFFERENT BUSINESS TYPES

\begin{tabular}{c|c|c|c|c}
\hline $\begin{array}{c}\text { Business } \\
\text { Type }\end{array}$ & Delay & $\begin{array}{c}\text { Packet } \\
\text { Lose }\end{array}$ & Jitter & Rate \\
\hline Conversational & $\mathrm{H}$ & $\mathrm{L}$ & $\mathrm{H}$ & $\mathrm{L}$ \\
\hline Streaming & $\mathrm{L}$ & $\mathrm{M}$ & $\mathrm{H}$ & $\mathrm{H}$ \\
\hline Interactive & $\mathrm{H}$ & $\mathrm{H}$ & $\mathrm{L}$ & $\mathrm{M}$ \\
\hline Background & $\mathrm{L}$ & $\mathrm{H}$ & $\mathrm{L}$ & $\mathrm{M}$ \\
\hline
\end{tabular}

Step 4: Calculated the entropy of each decision attribute $\mathrm{j}$ according to the definition of entropy, that is $e_{j}$.

$$
e_{j}=-\sum_{i=1}^{m} p_{i j} \cdot \ln p_{i j}, j=1,2, \cdots, n
$$

Where

$$
p_{i j}=a_{i j} / \sum_{i=1}^{m} a_{i j}, i=1,2, \cdots, m, j=1,2, \cdots, n .
$$

Step 5: Calculated the entropy weight of decision attributes based on entropy obtained by step 4 , and treated it as an objective weight $\omega_{j}^{O}$,

$$
\omega_{j}^{O}=\left(1-e_{j}\right) /\left(n-\sum_{j=1}^{n} e_{j}\right), j=1,2, \cdots, n
$$

Entropy represented the information that provided by decision attributes, the greater the entropy of decision attributes is, the more information it provides, if the entropy of a decision attribute is 0 , it indicates that the decision attribute does not provide any useful information for decision-making, all candidate networks have no difference, the decision attribute should be removed from the evaluation system.

Step 6: Combining the normalized subjective weights $\omega_{j}^{S}$ with the objective weights $\omega_{j}^{O}$, to get the integrated weights $\omega_{j}$ of decision attributes,

$$
\omega_{j}=\alpha \omega_{j}^{S}+(1-\alpha) \omega_{j}^{O}, j=1,2, \cdots, n
$$

Where, $\alpha \in(0,1)$, typical value is $\alpha=0.5$.

Step 7: Constructed a weighted normalized decision matrix $\mathbf{V}$,

$$
\mathbf{V}=\left[\begin{array}{cccc}
v_{11} & v_{12} & \cdots & v_{1 n} \\
v_{21} & v_{22} & \cdots & v_{2 n} \\
\vdots & \vdots & \ddots & \vdots \\
v_{m 1} & v_{m 2} & \cdots & v_{m n}
\end{array}\right]
$$

Where, $v_{i j}=a_{i j} \times \omega_{j}, i=1,2, \cdots, m ; j=1,2, \cdots, n$.

Step 8: To Determine the ideal solution $I^{+}$and the antiideal solution $I^{-}$,

$$
I^{+}=\left[v_{1}^{+}, v_{2}^{+}, \cdots, v_{m}^{+}\right], I^{-}=\left[v_{1}^{-}, v_{2}^{-}, \cdots, v_{m}^{-}\right]
$$

For desirable criteria,

$$
\begin{array}{r}
v_{i}^{+}=\max \left\{v_{i j}\right\}, v_{i}^{-}=\min \left\{v_{i j}\right\}, \\
i=1,2, \cdots, m ; j=1,2, \cdots, n
\end{array}
$$

For undesirable criteria,

$$
\begin{array}{r}
v_{i}^{+}=\min \left\{v_{i j}\right\}, v_{i}^{-}=\max \left\{v_{i j}\right\}, \\
i=1,2, \cdots, m ; j=1,2, \cdots, n
\end{array}
$$

Step 9: Calculated the absolute distance $S_{i}^{+}$between candidate network $i$ and the positive ideal solution $\mathrm{I}^{+}$, and calculated the absolute distance $S_{i}^{-}$between candidate network $\mathrm{i}$ and the negative ideal solution $I^{-}$,

$$
\begin{aligned}
& S_{i}^{+}=\sum_{j=1}^{n}\left|v_{i}^{+}-v_{i j}\right|, \\
& S_{i}^{-}=\sum_{j=1}^{n}\left|v_{i j}-v_{i}^{-}\right|, i=1,2, \cdots, m
\end{aligned}
$$

Step 10: Selected the network with the smallest value $S_{i}^{+}$and the largest value $S_{i}^{-}$as an ideal candidate network $\mathrm{P}$, namely

$$
P \sim\left(\min \left\{S_{i}^{+}\right\}, \max \left\{S_{i}^{-}\right\}, i=1,2, \cdots, m\right)
$$

Step 11: Calculated the effective distance $C_{i}$ between the candidate network $\mathrm{i}$ and the ideal network $\mathrm{P}$,

$$
\begin{gathered}
C_{i}=\sqrt{\left(S_{i}^{+}-\min \left\{S_{i}^{+}\right\}\right)^{2}+\left(\min \left\{S_{i}^{-}\right\}-S_{i}^{-}\right)^{2}}, \\
i=1,2, \cdots, m
\end{gathered}
$$

Rank each candidate network in descending order according to the value $C_{i}$. 


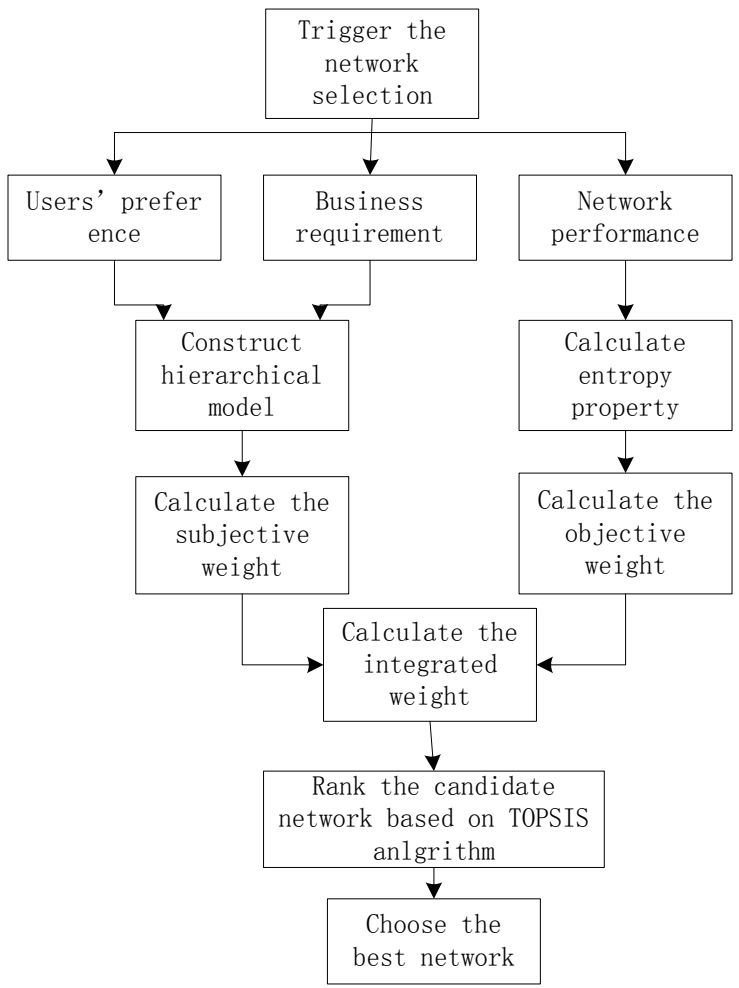

Figure 3. Algorithm flow

Step 12: Chose the network, which is the nearest to $C_{i}$, as the best network.

According to the above steps, the integrated weighting network access selection algorithm flow is shown in Fig. 3.

\section{SIMULATION AND ANALYSIS}

In order to evaluate the performance of the proposed integrated weighting network access selection algorithm, all initial network performance parameters in heterogeneous multi-cognitive wireless networks coexistence environment, namely the decision matrix elements are shown in TABLE 2, where the network load changing with the number of users. Assuming that the network is vacant at the beginning, the initial load is $100 \%$.

TABLE II. THE INITIAL PERFORMANCE PARAMETERS OF CANDIDATE NETWORKS

\begin{tabular}{c|c|c|c|c|c|c}
\hline & $\begin{array}{c}\text { Delay } \\
(\mathbf{m s})\end{array}$ & $\begin{array}{c}\text { Packet } \\
\text { Lose } \\
(\boldsymbol{\%})\end{array}$ & $\begin{array}{c}\text { Jitter } \\
(\mathbf{m s})\end{array}$ & $\begin{array}{c}\text { Rate } \\
(\mathbf{M b p s})\end{array}$ & $\begin{array}{c}\text { Load } \\
(\boldsymbol{\%})\end{array}$ & $\begin{array}{c}\text { Cost } \\
(\text { Yuan })\end{array}$ \\
\hline WRAN & 20 & 0.03 & 10 & 20 & 100 & 2.5 \\
\hline IIGN & 11 & 0.02 & 6 & 7 & 100 & 4 \\
\hline WLAN1 & 25 & 0.05 & 13 & 13 & 100 & 1.2 \\
\hline WLAN2 & 30 & 0.03 & 15 & 28 & 100 & 2 \\
\hline
\end{tabular}

Generally terminal support four kinds of business types that are conversational, streaming media, interactive and background, the relationship of decision attribute in different businesses are shown in TABLE 3. The data in the table subjectively is proposed by the author according to the experience. It is only intended to clarify the thought of method. Readers can adjust the values according to real situations.
TABLE III. THE RELATIONSHIP OF DECISION ATTRIBUTE IN DIFFERENT BUSINESSES

\begin{tabular}{|c|c|c|c|c|c|c|}
\hline Con versational & Delay & $\begin{array}{c}\text { Packet } \\
\text { Lose }\end{array}$ & Jitter & Rate & Load & Cost \\
\hline Delay & 1 & 4 & 1 & 3 & 5 & 6 \\
\hline Packet Lose & $1 / 4$ & 1 & $1 / 4$ & $1 / 2$ & $3 / 2$ & 2 \\
\hline Jitter & 1 & 4 & 1 & 3 & 5 & 6 \\
\hline Rate & $1 / 3$ & 2 & $1 / 3$ & 1 & 2 & 3 \\
\hline Load & $1 / 5$ & $2 / 3$ & $1 / 5$ & $1 / 2$ & 1 & 2 \\
\hline Cost & $1 / 6$ & $1 / 2$ & $1 / 6$ & $1 / 3$ & $1 / 2$ & 1 \\
\hline Streaming & Delay & $\begin{array}{l}\text { Packet } \\
\text { Lose }\end{array}$ & Jitter & Rate & Load & Cost \\
\hline Delay & 1 & $1 / 4$ & $1 / 6$ & $1 / 6$ & $1 / 4$ & $1 / 3$ \\
\hline Packet Lose & 4 & 1 & $1 / 2$ & $1 / 2$ & 2 & 2 \\
\hline Jitter & 6 & 2 & 1 & 1 & 2 & 3 \\
\hline Rate & 6 & 2 & 1 & 1 & 2 & 3 \\
\hline Load & 4 & $1 / 2$ & $1 / 2$ & $1 / 2$ & 1 & 2 \\
\hline Cost & 3 & $1 / 2$ & $1 / 3$ & $1 / 3$ & $1 / 2$ & 1 \\
\hline Interactive & Delay & $\begin{array}{c}\text { Packet } \\
\text { Lose }\end{array}$ & Jitter & Rate & Load & Cost \\
\hline Delay & 1 & $2 / 3$ & 8 & 2 & 2 & 4 \\
\hline Packet Lose & $3 / 2$ & 1 & 8 & 3 & 3 & 4 \\
\hline Jitter & $1 / 8$ & $1 / 8$ & 1 & $1 / 4$ & $1 / 4$ & $1 / 2$ \\
\hline Rate & $1 / 2$ & $1 / 3$ & 4 & 1 & 2 & 3 \\
\hline Load & $1 / 2$ & $1 / 3$ & 4 & $1 / 2$ & 1 & 2 \\
\hline Cost & $1 / 4$ & $1 / 4$ & 2 & $1 / 3$ & $1 / 2$ & 1 \\
\hline Background & Delay & $\begin{array}{l}\text { Packet } \\
\text { Lose }\end{array}$ & Jitter & Rate & Load & Cost \\
\hline Delay & 1 & $1 / 3$ & $3 / 2$ & $1 / 5$ & $1 / 2$ & $1 / 2$ \\
\hline Packet Lose & 3 & 1 & 5 & $1 / 2$ & 2 & 3 \\
\hline Jitter & $2 / 3$ & $1 / 5$ & 1 & $1 / 6$ & $1 / 3$ & $1 / 2$ \\
\hline Rate & 5 & 2 & 6 & 1 & 2 & 4 \\
\hline Load & 2 & $1 / 2$ & 3 & $1 / 2$ & 1 & $3 / 2$ \\
\hline Cost & 2 & $1 / 3$ & 2 & $1 / 4$ & $2 / 3$ & 1 \\
\hline
\end{tabular}

Calculating the normalized subjective weights:

1) Conversational:

$W^{S}=\left[\begin{array}{llllll}0.3347 & 0.0846 & 0.3347 & 0.1317 & 0.0686 & 0.0457\end{array}\right]$

2) Streaming :

$W^{S}=\left[\begin{array}{llllll}0.0396 & 0.1729 & 0.2798 & 0.2798 & 0.1372 & 0.0907\end{array}\right]$

3) Interactive :

$W^{S}=\left[\begin{array}{llllll}0.2674 & 0.3504 & 0.0358 & 0.1606 & 0.1190 & 0.0668\end{array}\right]$

4) Background:

$W^{S}=\left[\begin{array}{llllll}0.0717 & 0.2501 & 0.0522 & 0.3710 & 0.1518 & 0.1032\end{array}\right]$

Calculating the entropy of each decision property based on the step 4, and calculating the objective weights based on step 5 , then calculating the integrated weights of each business decision based on step 6 .

1) Conversational:

$W=\left[\begin{array}{llllll}0.2472 & 0.1173 & 0.2378 & 0.2193 & 0.0343 & 0.1442\end{array}\right]$

2) Streaming :

$W=\left[\begin{array}{llllll}0.0996 & 0.1614 & 0.2103 & 0.2934 & 0.0686 & 0.1667\end{array}\right]$

3) Interactive :

$W=\left[\begin{array}{llllll}0.2135 & 0.2501 & 0.0883 & 0.2338 & 0.0596 & 0.1547\end{array}\right]$

4) Background:

$W=\left[\begin{array}{llllll}0.1156 & 0.2001 & 0.0965 & 0.3390 & 0.0759 & 0.1729\end{array}\right]$

In order to fully evaluate the performance of this algorithm, simulation was analyzed in the independent business scenario and random scenario. 


\section{A. Independent business scenario}

Conversation business, for example, assumed all users with the same business scenario.

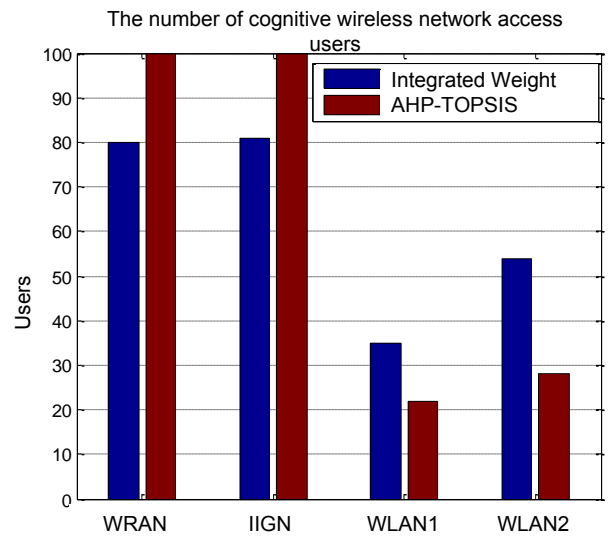

Figure 4. The distribution of accessed users in conversational business scenario

Fig. 4 shows that when the number of users is 250 , this algorithm (referred to as integrated weight algorithm) and the algorithm (called AHP-TOPSIS algorith m) [15] were used to performing network selection, and then the distribution of accessed users is obvious. The figure shows that after performing AHP-TOPSIS algorithm, a large number of users access to WRAN and IIGN network with a network congestion, but WLAN1 and WLAN2 loads are very low. When performing the integrated algorithm, WLAN1 and WLAN2 share more loads, reducing congestion of IIGN and WRAN. Because the algorithm introduced entropy as objective weight, it can effectively balance the load and achieve coordinated allocation of resources. Comparison result shows the AHP-TOPSIS algorithm weights of each decision attribute are fixed, which can not balance the loads in time.

Fig. 5 shows the relationship between load entropy and the number of accessed users in conversation business scenario. The figure shows that with the increasing of access users, the load entropy of each network increased, which meant that the importance of the load to the six kinds of decision attributes is increased, the probability of user selects the low network load is increased, the business stream separation has been formed. After the separation of business stream for some time, the differences between the networks will be decreased gradually, and the load entropy will be gradually stabilized.

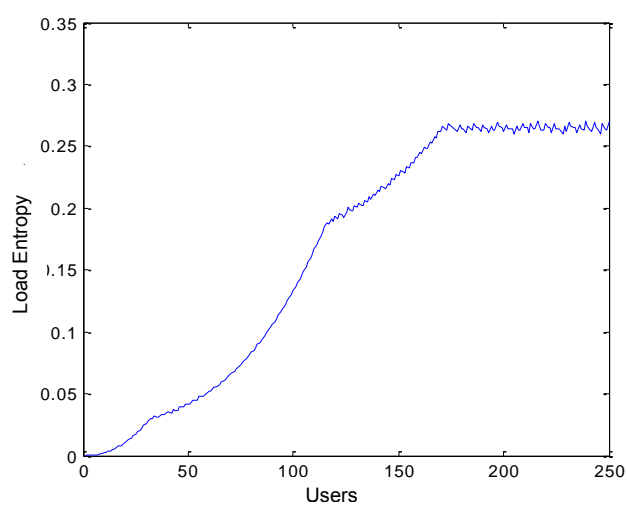

Figure 5. The relationship between load entropy and the number of accessed users
Further analys is for the independent business streaming media, interactive class and background class, the distribution of accessed users and the relationship between load entropy and the number of accessed users, which are similar with the independent conversation business.

\section{B. Random business scenario}

In the random business scenario, each user generates their own independent business randomly, and then starts the network selection algorithm to perform optimal network selection.

Fig. 6 shows each network access rate after executing integrated weighting network access selection algorithm and AHP-TOPSIS algorith $m$ in random business scenario. The comparis on results show that since the initial moment of each network are in an idle state, no matter what kind of service the user requests, the results of the two algorithms are similar. But with the number of accessed users increasing, the advantages network performance parameters began to change, users began to access other networks. At this stage, because the integrated weighting network access selection algorithm takes into account the actual network load changes, the weight calculation by adding the objective entropy, when the integrated weighting network access selection algorithm is executed, the probability curve of each selected network converged faster, the selected rate of the four networks are similar, and the loads are more balanced.

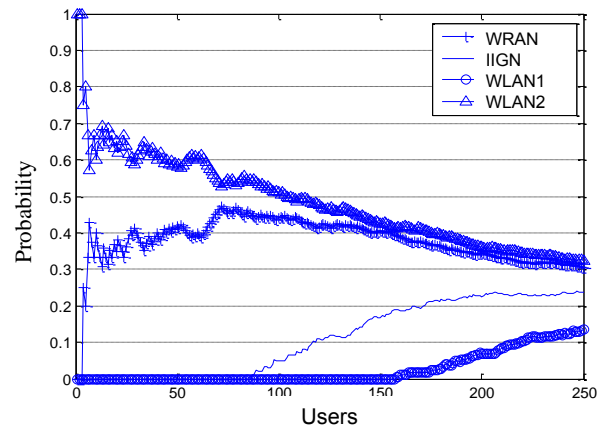

(a) Integrated Weight Algorithm

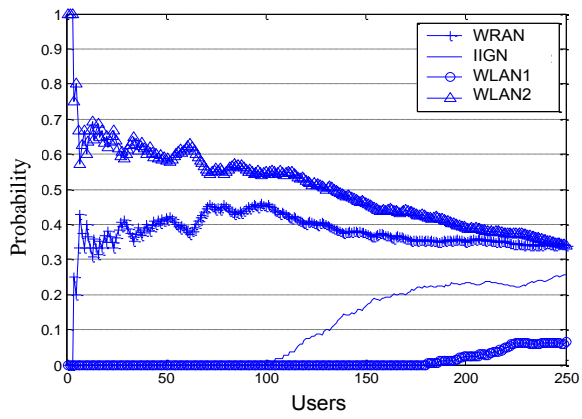

(b) AHP-TOPSIS Algorithm

Figure 6. Network access probability in random business scenario

\section{CONCLUSIONS}

Considering the three factors that user's preference, business requirement and network performance, this paper presented an integrated weighting network access selection algorithm under heterogeneous multi-cognitive wireless network coexistence environment. The algorithm based on AHP-TOPSIS algorithm which defined positive and 
negative ideal values, and used the distance value between the candidate networks to avoid the ranking abnormality problem when executing the TOPSIS algorithm. The algorithm introduced entropy as objective weight for decision attributes, to avoid the subjective and arbitrary problem when performing the AHP algorithm. And the algorithm used the relative relationship of the decision attributes, business types, and the network performance to define the subjective weight, then combined the objective weight with the subjective weight to form an integrated weight. Adopting the algorithm to execute optimal access network selection, can balance network loads, and use network resources reasonably.

The simulation results show that in independent business scenario, the algorithm can balance the load efficiently, in random scenario, with the number of accessed users increasing, the probability curve of each network selected convergent fast, the selected rate of the four networks are similar, and the loads are more balanced. Concluded that the proposed integrated weighting network access selection algorithm can select candidate network correctly depending on the different business needs in the heterogeneous multi-cognitive wireless network coexistence environment, and can meet the service quality while achieving the entire network load balancing, while improving resource utilization.

\section{ACKNOWLEDGMENT}

Thanks for these foundation item: National Natural Science Foundation of China (No.60872038 ); Natural Science Foundation of Hainan Province(No. 614237)

\section{REFERENCES}

[1] Akyildiz I F. A survey on spectrum management in cognitive radio net works[J]. IEEE Communicat ions Magazine, 2008, 46(4):40 - 48

[2] IEEE Std 802.22-2011. IEEE Standard for Information Technology Telecommunications and information exchange between systems Wireless Regional Area Net works (WRAN)-Specific requirementsPart 22: Cognitive Wireless RAN Medium Access Control (MAC) and Physical Layer (PHY) specifications: Policies and procedures for operation in the TVbands[S], 2011: 1-672.

[3] IEEE Std 802.11af. IEEE Standard for Information Technology Telecommunications and information exchange between systems Local and metropolitan area networks Specific requirements-Part 11: Wireless LAN Medium Access Control (MAC) and Physical Layer (PHY) Specifications-Amendment: TV White Spaces Operation[S], 2009.
[4] Std ECMA-392. Standard ECMA 392 MAC and PHY for Operation in TVWhite Space[S], 2012: 1-190.

[5] Xu P, Fang X, Liu X. A Non-cooperat ive Pairwise Matrices Game Model for Heterogeneous Network Selection[C]// 2010 International Conference on Communications and Mobile Computing. IEEEComputer Society, 2010:387-391.

[6] Niyato D, Hossain E. Dynamics of Network Selection in Heterogeneous Wireless Networks: An Evolutionary Game Approach[J]. IEEE Transactions on Vehicular Technology, 2009, 58(4):2008-2017.

[7] Gu C, Song M, Zhang Y, et al. Novel Network Selection Mechanism Using AHP and Enhanced GA[C]// Communication Networks and Services Research Conference, 2009. CNSR '09. Seventh Annual. IEEE, 2009:397-401.

[8] Lahby M, Cherkaoui L, Adib A. Network selection algorithm based on Diff-AHP and TOPSIS in heterogeneous wireless networks[C]// 2012 International Conference on Multimedia Computing and Systems. 2012:485 - 490.

[9] Goel N, Purohit N, R. Singh B. A New Scheme for Network Selection in Heterogeneous Wireless Network using Fuzzy Logic $[J]$. International Journal of Computer Applications, 2014, 88(3):1-5.

[10] Trestian R, Ormond O, Muntean G M. Enhanced Power-Friendly Access Net work Selection Strategy for Multimedia Delivery Over Heterogeneous Wireless Networks[J]. Broadcasting IEEE Transactions on, 2014,60(1):85 - 101.

[11] Du Z, Wu Q, Yang P. Dynamic User Demand Driven Online Network Selection[J]. Communications Letters IEEE, 2014, 18(3):419-422

[12] Sgora A, Vergados D D, Chatzimisios P. An Access Network Selection Algorithm For Heterogeneous Wireless Environments[C]// Computers and Communications (ISCC), 2010 IEEE Symposium on. 2010:890-892.

[13] Sun Y, Liu C, Yang P, et al. A smart vertical handoff decision algorithm based on queuing theory[C]// Adv anced Communication Technology (ICACT), 2014 16th International Conference on. IEEE, 2014:1217-1222.

[14] Trestian R, Ormond O, Muntean G M. Enhanced Power-Friendly Access Network Selection Strategy for Multimedia Delivery Over Heterogeneous Wireless Networks[J]. Broadcasting IEEE Transactions on, 2014,60(1):85 - 101.

[15] Bakmaz B, Bojkovic Z, Bakmaz M. Traffic parameters influences on network selection in heterogeneous wireless environment [C]. //Systems, Signals and Image Processing (IWSSIP), 2012 19th International Conference on. IEEE, 2012:292-295.

[16] Jacob J S, Preetha K G. A QoS and QoE collaborative scheme for effective network selection in $4 \mathrm{G}$ communication systems[C]// Recent Advances in Computing and Software Systems (RACSS), 2012 International Conference on. IEEE, 2012:170-173. 